A RTI G O

\title{
ON \\ EsTATINAS E O FígAdo: \\ Mito ou Realidade
}

\section{Autores}

Angelo Alves de Mattos*

Ângelo Zambam de Mattos**

*Professor Titular da Disciplina de Gastroenterologia e do Curso de Pós-Graduaçáo em Hepatologia da Universidade Federal de Ciências da Saúde de Porto Alegre (UFCSPA).

**Professor Adjunto de Gastroenterologia e do Curso de Pós-Graduação em Hepatologia da UFCSPA. Mestre e Doutor em Hepatologia.

Endereço para correspondência:

Angelo Alves de Mattos

Rua Coronel Aurelio Biencourt 35, apart. 201

Porto Alegre/RS

E-mail:angeloamattos@gmail.com

\section{RESUMO}

Os autores após contextualizarem a importância das doenças hepáticas crônicas avançadas, uma vez que ocupam o oitavo lugar no ranking de mortalidade no Brasil, ao abordarem a classificaçáo da cirrose, ressaltam a importância da hipertensão porta clinicamente significativa (HPCS). Sendo ela o fator preponderante não só do surgimento das varizes gastroesofágicas, mas também das demais complicaçóes da cirrose trazem reflexôes em relaçáo a uma nova postura a ser tomada. Assim, o objetivo do tratamento não seria a profilaxia do sangramento das varizes mas a profilaxia/tratamento da HPCS. Ao fazerem esta assertiva, demonstram a importância do tratamento da doença hepática de base bem como da hipertensão porta com os ß-bloqueadores não específicos. Tendo em vista, estas medidas não terem a efetividade ideal, fazem reflexóes em relação ao papel das estatinas no tratamento das doenças hepáticas. Ao salientarem seu papel antioxidante; anti-inflamatório; antitrombótico; antifibrótico e na melhora da disfunção endotelial, apresentam evidências de sua utilização como arma terapêutica no tratamento dos pacientes com cirrose e HPCS. No entanto, ao ressaltarem o papel das estatinas no armamentário terapêutico, julgam ainda necessária a existência de mais estudos prospectivos randomizados para que seja tomada uma conduta mais definitiva. 
Quando avaliamos o impacto das doenças no Brasil, observamos que as doenças do fígado ocupam o oitavo lugar no ranking de mortalidade. A principal causa de hospitalização e de óbito por doenças hepáticas é a cirrose $^{1}$. Ao avaliarmos o burden da doença hepática crônica no mundo ${ }^{2}$, é projetado um acometimento de 1,5 bilhão de pacientes, sendo estimado que as hepatopatias crônicas sejam responsáveis por 2.000 .000 mortes ao ano, número este provavelmente subestimado.

Também é importante que seja avaliada os estágios clínicos da classificação da cirrose $^{3}$. No estágio 0 observamos doença compensada sem hipertensão porta clinicamente significativa - HPCS (gradiente de pressão venoso hepático - GPVH $>5 \mathrm{mmHg}$ e $<$ $10 \mathrm{mmHg}$ ) e com boa resposta ao tratamento etiológico; no estágio 1 a doença permanece compensada e sem a presença de varizes gastroesofágicas (VGE), no entanto, com HPCS $(\mathrm{GPVH}>10 \mathrm{mmHg}$ ) e logo com alto risco de desenvolver varizes, carcinoma hepatocelular (CHC) e de descompensaçáo e quando no estágio 2 podemos observar o surgimento de varizes (mortalidade em 5 anos de $10 \%$ se náo houver descompensação). No estágio 3 já é constatado hemorragia digestiva alta (HDA) por ruptura de varizes, sendo a mortalidade em 5 anos de $20 \%$ se náo houver descompensação da hepatopatia; no estágio 4 observamos o primeiro episódio de decompensaçáo, propriamente dita, (sem considerar sangramento, mais frequentemente com o surgimento de ascite) com uma mortalidade em 5 anos de 55-80\% e no estágio 5 temos episódios futuros de descompensaçáo com uma mortalidade que pode alcançar $90 \%$ em 5 anos. Finalmente, no estágio 6 temos um paciente com cirrose descompensada de forma avançada (ascite refratária, infecção, encefalopatia hepática $(\mathrm{EH})$ persistente, icterícia e disfunção renal). Como podemos observar o prognóstico da doença começa a comprometer a sobrevida do paciente de forma mais significativa à partir do estágio 3 , quando do sangramento por ruptura de VGE, mas certamente se torna mais evidente no estágio 4, onde há um "pulo" significativo na mortalidade. Desta forma, é racional termos como premissa não só evitar o sangramento mas também as demais manifestaçóes que ocorrem nos pacientes com cirrose e que, a HPCS é o principal ator destas complicações.

Em linhas gerais, é relevante o conhecimento de que as varizes estáo presentes em até $40 \%$ dos pacientes com cirrose compensada (Child A) e em até $85 \%$ quando a doença descompensa (Child C)4. Quando avaliamos uma coorte de pacientes com doença hepática crônica em nível ambulatorial, o sangramento digestivo foi a segunda complicação mais frequente na apresentação destes doentes, principalmente quando a etiologia da doença hepática esteve relacionada ao álcool ${ }^{5}$. Ressaltamos que a recorrência do sangramento em um ano pode ser de até de $60 \%$ e que a mortalidade atual de cada 
sangramento varia de 15-20\%. Também chamamos atenção de que pacientes com varizes de médio/grosso calibre tem risco de sangramento de 25-30\% em dois anos ${ }^{4}$.

Desta forma, com o intuito de evitar o sangramento, se torna mister a realização da profilaxia primária da HDA por ruptura de varizes. Até há pouco, os critérios de screening para a pesquisa de varizes eram baseados na realização da endoscopia digestiva alta no momento do diagnóstico da cirrose. Mais recentemente, o consenso de Baveno VI, realizado em $2015^{6}$, recomendou que, naqueles pacientes que realizassem elastografia e se observasse no Fibroscan ${ }^{\circledast}$ uma medida inferior a $20 \mathrm{KPa}$, em dois procedimentos realizados em dias distintos, bem como plaquetas em níveis superiores a $150.000 / \mathrm{mm}^{3}$, estaria dispensada a endoscopia, desde que a etiologia da hepatopatia fosse viral. $\mathrm{O}$ Guidance da American Association for the Study of Liver Diseases (AASLD), publicado mais recentemente ${ }^{7}$, sugere que, se os parâmetros anteriormente referidos fossem atendidos, seria baixa (inferior a 5\%) a possibilidade de varizes de alto risco para o sangramento e que esta postura evitaria a realização de 20-25\% das endoscopias. As últimas recomendações da Sociedade Brasileira de Hepatologia (SBH) consideram tanto a elastografia quanto a endoscopia válida no screening dos pacientes ${ }^{8}$. Este também tem sido o nosso posicionamento 9 .
Embora cresça cada vez mais os esforços na realização de testes não invasivos para avaliar a presença de VGE de risco, a European Association for the Study of the Liver (EASL ${ }^{10}$ enfatiza que os mesmos estão restritos a doença compensada e, tendo em vista o alto risco das VGE em pacientes com doença descompensada, a endoscopia deve ser sempre realizada nestes pacientes para avaliar o tamanho e a presença de red wale marks nas varizes.

Assim, poderia ser considerado que, no screening e surveillance das $\mathrm{VGE}^{11}$, como na doença compensada a prevalência de VGE é de 30-40\% e que somente 10-20\% apresentam varizes de risco, seria válido os métodos não invasivos, uma vez que caso os dados sejam negativos a chance de VGE de risco é $<5 \%$ e se evitaria até $30 \%$ de endoscopias. Já na doença descompensada todos deveria fazer endoscopia anual.

Uma vez feitas estas consideraçóes, no que tange especificamente à profilaxia, quando considerada a assim chamada profilaxia pré-primária, mais recentemente, na doença crônica compensada sem HPCS, a meta a ser considerada seria evitar o seu desenvolvimento com o intuito de prevenir o surgimento de varizes, e também as demais complicaçôes da hipertensão portal (ascite, EH...) ${ }^{12}$. Para tanto dever-se-ia tratar a causa da cirrose e adequar estilo de vida do paciente.

Ao ser avaliado o benefício dos análogos nucleos(t)ídios no tratamento dos doentes 
com cirrose pelo vírus da hepatite B (VHB), estudo prospectivo de 12 anos $^{13}$, demonstrou regressão significativa das VGE, quando existentes, e baixo risco no desenvolvimento de varizes.

Quando avaliados os efeitos hemodinâmicos da resposta virológica sustentada (RVS) em 226 pacientes com cirrose decorrente ao vírus da hepatite $\mathrm{C}$ (VHC) e HPCS, em estudo prospectivo e multicêntrico ${ }^{14}$, foi observado haver diminuição do GPVH porém a HPCS persistiu em 78\% dos casos, permanecendo assim o risco de descompensação. Mais recentemente ${ }^{15}$ quando avaliado o impacto da RVS nas VGE em 230 pacientes com cirrose pelo VHC, estudo multicêntrico avaliando 151 pacientes com cirrose e RVS, daqueles que não tinham varizes $12,5 \%$ as desenvolveram, mesmo após a RVS, e dos que tinham VGE de baixo risco, $24 \%$ evoluíram para varizes de alto risco, mostrando que um tratamento exitoso não impede o desenvolvimento e a progressão das varizes. No mesmo ano, foi publicado na Espanha ${ }^{16}$ estudo avaliando o papel da RVS a longo prazo nos pacientes com cirrose e HPCS demonstrando a sua permanência em mais de $50 \%$ dos casos, sendo que embora houvesse diminuição da rigidez hepática, os achados da elastografia não se relacionavam com as alteraçóes do GPVH.

Da mesma forma, embora mudanças no estilo de vida diminuam a hipertensão porta em cirróticos obesos, não evitam a $\mathrm{HPCS}^{17}$.
Desta forma, podemos depreender que outras medidas se fazem necessárias, principalmente quando houver HPCS, uma vez que esta parece ser a balizadora dos episódios de descompensação.

Baseados no estudo de Groszmann et al. ${ }^{18}$, não se considerava o papel dos B-bloqueadores em pacientes com cirrose sem varizes. Seu uso ficava indicado em pacientes com varizes pequenas, principalmente quando fossem observadas manchas vermelhas nas mesmas ou quando estivermos frente a uma doença hepática avançada. Ressaltese ser fundamental a sua utilização em pacientes com varizes médias/calibrosas ${ }^{6,8}$. Nesta situação é discutido o papel da ligadura elástica, havendo autores que preconizam seu uso em detrimento da terapia farmacológica, no entanto, esta é uma conduta a nosso ver controversa, não sendo objeto da presente revisão.

Mais de 10 anos após o estudo de Grozmann et al. ${ }^{18}$ foi revisitado o desenvolvimento da circulação hiperdinâmica na cirrose e a resposta aos $\beta$-bloqueadores ${ }^{19}$, tendo os autores demonstrado que paciente sem HPCS apresentam menos circulação hiperdinâmica e menor redução da hipertensão porta com o uso de ß-bloqueadores, quando comparados aos pacientes com HPCS e que o estudo anterior não havia separado estas duas populaçôes de pacientes.

Tendo em vista estes achados foi desenhado o estudo PREDESCI, que avaliava 
o papel dos ß-bloqueadores em pacientes com $\mathrm{HPCS}^{20}$. Este foi um estudo randomizado, prospectivo, controlado, multicêntrico e duplo cego em pacientes com cirrose compensada e HPCS. Os pacientes que responderam agudamente aos ß-bloqueadores foram então randomizados em um grupo com propranolol vs. placebo e aqueles que não haviam respondido a carvedilol vs. placebo. Dos 210 pacientes randomizados (100 propranolol ou carvedilol vs. 101 placebo), descompensação/óbito ocorreram, respectivamente, em $16 \%$ vs. $27 \%$; $\mathrm{p}=0,041$. A diferença mais importante esteve relacionada a incidência de ascite. Concluíram então os autores que os ß-bloqueadores aumentam a sobrevida livre de descompensação (enaltecendo o papel da menor incidência de ascite).

A despeito de eventuais limitaçôes metodológicas (a maior parte dos pacientes tinham cirrose relacionada ao $\mathrm{VHC}$ e não haviam sido tratados), acreditamos que este estudo é um marco no tratamento dos pacientes cirrose. A partir de então, ao nos depararmos com um paciente com doença hepática crônica avançada nosso olhar deve estar voltado ao tratamento da HPCS e não mais pontualmente ao tratamento das VGE.

Desde a última reunião de Baveno ${ }^{6}$ o carvedilol já havia sido incorporado como droga de primeira linha na profilaxia primária.

Os ß-bloqueadores não seletivos (propranolol)são utilizados no tratamento da hipertensão portal há 4 décadas $^{21}$. $\mathrm{O}$ propra- nolol atua bloqueando os receptores adrenérgicos $ß 1$ e $ß 2$, com consequente diminuição do débito cardíaco e ação no sistema vascular esplâncnico. Já o carvedilol é um ßbloqueador não seletivo, com atividade intrínseca anti-a1-adrenégica, que atua também diminuindo a resistência vascular intrahepática e da circulação colateral porta, sendo mais efetivo em diminuir a pressáo no sistema porta do que o propranolol ${ }^{12}$.

Baixar a pressáo porta melhora a evolução da cirrose, na presença ou ausência de ascite, conforme demonstrada na recente metanálise realizada com 15 estudos e mais de 1100 pacientes $^{22}$. Quando avaliada a profilaxia primária, ao redor da metade dos pacientes respondedores aos ß-bloqueadores além de terem apresentado menos sangramento decorrente da ruptura de varizes, apresentaram menos complicaçóes decorrentes da descompensação da cirrose, menor necessidade de transplante hepático e menor mortalidade.

Qual o mais eficaz ß-bloqueadores não seletivo (propranolol vs. carvedilol) a ser utilizado, a despeito das metanálises realizadas $^{23,24}$, ainda náo encontra uma resposta definitiva na literatura, haja visto não haver estudos comparativos head-to-head entre os dois fármacos. No entanto, há uma tendência a utilizaçáo do carvedilol, por se acreditar em sua maior atuação em baixar o GPVH (deva ser ressaltado que pode levar a hipotensão, particularmente na cirrose descompensada) ${ }^{25}$. 
A despeito do que foi até então exposto, inclusive de uma proposta para atentar mais para a HPCS e menos para a presença de VGS, podemos concluir que mesmo com o tratamento da doença de base, bem como com a utilização das drogas vasoativas anteriormente citadas, uma parcela dos pacientes com cirrose não respondem do ponto de vista hemodinâmico a terapia farmacológica. $\mathrm{O}$ ideal seria identificar estes pacientes medindo o GPVH, método invasivo, pouco acessível na maior parte dos centros, tornando-se assim, uma conduta assistencial de pouca valia. Por outro lado, a elastografia hepática, um método não invasivo, não retrata a real presença de HPCS com a queda do GPVH para que possamos utilizá-la para individualizar estes pacientes. Desta forma, uma opção seria a introdução de um outro fármaco que pudesse atuar ao nível da pressão porta, somando efeitos às medidas terapêuticas anteriormente referidas. A este respeito, muito se tem estudado em relação as estatinas.

As estatinas são inibidores da hydroxymethylglutaryl-CoA (HMG CoA) reductase que diminuem de forma significativa o colesterol total e o LDL. Introduzida em 1987 (lovastatina) para diminuir o colesterol, reduz eventos cardiovasculares e diminui a mortalidade destes pacientes. Podemos inferir a existência de 1 bilhão de potenciais usuários ${ }^{26}$.

Dentre os efeitos pleiotrópicos das estatinas salientamos seu papel antioxidante; anti-inflamatório; antitrombótico; antifibrótico e uma ação na melhora da disfunção endotelial. Tem sido utilizada em uma plêiade de doenças: câncer; septicemia severa; doença bronco pulmonar obstrutiva crônica; esclerose múltipla; doença de Parkinson, doença de Alzheimer; doença renal crônica..., embora os resultados sejam provenientes de estudos observacionais não confirmados em estudos prospectivos, controlados e randomizados. Sua indicação somente foi confirmada para diminuir risco cardiovascular e baixar níveis de colesterol ${ }^{27}$.

Tendo em vista suas múltiplas ações as estatinas poderiam ser consideradas como uma nova indicação para os pacientes com cirrose $^{28}$. Assim, deveriam ser enaltecidas suas propriedades na redução na pressão porta (pelo aumento da produção de óxido nítrico intra-hepático, com diminuição do tônus vascular em pacientes com cirrose) ${ }^{29}$; melhora da disfunção endotelial sinusoidal e microvascular hepática; diminuição da fibrogênese; proteção contra lesão de isquemia/ reperfusão e prolongamento da preservação do enxerto; redução da sensibilidade a dano hepático mediado por endotoxina, prevenção $\mathrm{da}$ acute-on chronic liver failure (ACLF) e da lesão hepática após choque hipovolêmico; prevenção/retardamento da progressão da cirrose de qualquer etiologia; potenciais efeitos benéficos na progressáo de outras doenças hepáticas (colangite esclerosante) e potencial efeito na prevenção do $\mathrm{CHC}^{28}$. 
Esta droga, por aumentar a geração de óxido nítrico no fígado e melhorar a função endotelial, traduz uma melhora nos níveis de pressão porta, bem como na função e perfusão do fígado. Este fato foi demonstrado clinicamente em um estudo utilizando sinvastatina em pacientes com cirrose e GPVH maior do que $12 \mathrm{~mm}$ de $\mathrm{Hg}^{30}$.

Em um estudo retrospectivo, avaliando pacientes com cirrose pelo VHC, o uso das estatinas estava associado à diminuição (40\%) dos episódios de descompensação e da mortalidade. No entanto, os autores ainda não encontravam força de evidência para sua indicação na cirrose ${ }^{31}$.

$\mathrm{O}$ efeito da atorvastatina na pressão porta e na evolução clínica em pacientes com cirrose $\mathrm{e}^{32}$ foi mais recentemente avaliado em uma coorte de 23 pacientes consecutivos com cirrose, que foram randomizados em um grupo com propranolol $(n=12)$ vs. outro que utilizava atorvastatina $20 \mathrm{mg} /$ dia com propranolol $(n=11)$. O GPVH foi estimado no início do estudo e após 30 dias, e, os desfechos clínicos foram avaliados após 1 ano. A despeito da maior diminuição do gradiente de pressão no grupo em que o tratamento foi combinado, não houve diferença nos desfechos avaliados (sangramento; EH, número de paracenteses, peritonite bacteriana espontânea e morte). Como o número de pacientes foi muito pequeno se faz necessários estudos com números mais robustos de doentes.
Outro estudo, com desenho semelhante ao anterior, agora utilizando a sinvastatina e o carvedilol na profilaxia primária em pacientes com cirrose e GPVH maior do que $12 \mathrm{~mm}$ de $\mathrm{Hg}$ foi realizado ${ }^{33}$. A despeito do grupo randomizado ser maior (110 pacientes com carvedilol e 110 com terapia combinada) os autores não conseguiram demonstrar diferença na resposta hemodinâmica quando avaliada em 3 meses, bem como nos desfechos clínicos. É feita uma ressalva para que se deva atentar em relação aos efeitos adversos quando do uso em pacientes Child C.

Importante estudo apresentado na literatura foi relativo a adição de sinvastatina à terapia standart na profilaxia secundária dos pacientes com VGE (BLEPS trial) ${ }^{34}$. Embora a profilaxia secundária fuja do escopo da presente revisão, vale pequena consideração pela importância que julgo ter este estudo avaliando o papel das estatinas. Este é um estudo prospectivo, randomizado, multicêntrico e duplo cego com 158 pacientes com cirrose em profilaxia secundária (ß-bloqueador e ligadura elástica). Foi utilizado sinvastatina $(20 \mathrm{mg} / \mathrm{d}$ inicial, aumentando posteriormente a dose para 40 $\mathrm{mg} / \mathrm{d} ; \mathrm{n}=69)$ e comparado com um grupo placebo $(n=78)$. Foram observados, respectivamente, 6 (9\%) vs. 17 (22\%) óbitos; HR = 0.39; 95\% CI: 0.15-0.99; $\mathrm{p}=0,030$, não se tendo observado aumento na sobrevida em pacientes Child C. Não houve diferença no que tange a ressangramento ou a presença 
de eventos adversos, no entanto, dois pacientes com doença hepática avançada desenvolveram rabdomiólise. Concluem os autores que a adição de sinvastatina à terapia standard não diminui o ressangramento porém aumenta a sobrevida.

É interessante o conhecimento de que o efeito benéfico da estatina está diminuído nos carreadores da variante SLCO1B (polimorfismo de nucleotídeo de perda de função em SLCO1B1). A perda de função da variante p.A174V do transportador da estatina SLCO1B1 (um transportador de ânions orgânicos que regula a captação hepática de estatinas) — rs4149056 — leva a diminuição da função de transporte e, logo, de seu efeito. Também é relevante o fato de que o genótipo SLCO1B1 rs4149056 está associado a toxidade muscular, bem como ao fato de que a doença hepática alcoólica progressiva está associada a uma menor expressão do SLCO1B ${ }^{28,35,36,37}$.

O papel das estatinas na doença hepática gordurosa não alcoólica/esteatohepatite não alcoólica, diminuindo a fibrose e na colangite esclerosante primária levando a uma maior sobrevida, embora descritos, ainda necessitam de melhor avaliação ${ }^{28,38}$.

Em uma revisão sistemática e metanálise realizada avaliando o papel das estatinas na cirrose ${ }^{39}$, embora evidenciando diminuição de 46\% no risco de decompensação/ mortalidade, bem como uma diminuição de $27 \%$ no risco de sangramento ou pro- gressão da hipertensão porta, ainda não parece ser conclusivo, se fazendo necessários mais estudos prospectivos controlados e randomizados com placebo para uma tomada de decisão mais conclusiva.

Quando avaliado o papel das estatinas e o risco de $\mathrm{CHC}$ em pacientes com hepatite crônica viral ${ }^{40}$, em uma coorte de com 16.668 adultos, dos quais 8.334 utilizavam estatinas lipofílicas (sinvastatina e atorvastatina) e hidrofílicas (pravastatina e rosuvastatina) e 8.334 eram não usuários, foi observado que o risco de $\mathrm{CHC}$ em 10 anos foi significativamente menor entre os usuários das estatinas lipofílicas. No entanto, este estudo apresenta risco de viés, necessitando confirmação.

As reaçóes adversas mais relevantes com a utilização das estatinas, observadas nos pacientes com hepatopatia, são as hepáticas e a toxicidade muscular ${ }^{28}$. A lesão hepática é uma reação idiossincrática (mais comumente durante o $1^{\circ}$ mês). As estatinas mais comumente implicados são a atorvastatina, a sinvastatina e a fluvastatina. A maioria das elevações das enzimas hepáticas são transitórias e assintomáticas. Pacientes com doença hepática compensada parecem não ter um risco maior do que a população em geral, não havendo razão para restringir sua prescrição quando indicada. Quando avaliados os pacientes com cirrose descompensada pode haver aumento nas concentraçóes séricas dos medicamentos e subsequente maior risco de eventos adversos. 
Embora a rabdomiólise seja apontada como um efeito adverso importante $e^{41}$ é interessante lembrar a recente revisão sistemática e metanálise que avalia a segurança das estatinas na cirrose ${ }^{42}$. Este risco não está descrito com o uso de sinvastatina $20 \mathrm{mg} / \mathrm{d}$. O risco de rabdomiólise com sinvastatina $40 \mathrm{mg} / \mathrm{d}$ é de $2 \%$, quarenta vezes maior do que em não cirróticos. Não deve assim, ser utilizada em pacientes com cirrose descompensada. A estatina ideal a ser utilizada ainda não foi definida. Do ponto de vista de farmacodinâmica podemos observar muitos estudos com a sinvastatina em pacientes com cirrose. No entanto, ao avaliarmos a farmacocinética (PK), não conhecemos estudos a seu respeito. No que tange a artovastatina observa-se uma concentração máxima quatro vezes maior em pacientes com cirrose Child A e 16 vezes maior nos Child B. Já a rosuvastatina e a pitavastatina apresentam mínima alteração na $\mathrm{PK}$ em pacientes com cirrose Child A e estudo com doses repetidas de rosuvastatina, mostram alteração mínima da PK em pacientes Child A e B.

Enfim, podemos recomendar atualmente a utilização das estatinas na prática clínica para os pacientes com cirrose com o intuito precípuo de tratar aqueles com HPCS? A resposta seria não. A despeito do entusiasmo inicial, necessitamos de mais estudos prospectivos, randomizados, controlados e duplo cegos. Seu uso deve ser cauteloso nos pacientes com cirrose descompensada (não deveria ser utilizada em Child C), embora possa ser utilizada na cirrose compensada com intuito de diminuir o risco cardiovascular.

Vários estudos randomizados e controlados estáo em andamento. Assim temos o estudo SIMPRO: prevenção da progressão da HPCS em pacientes com cirrose compensada e GPVH entre 6-10 mmHg (NCT 01282398); SACRED: pacientes com cirrose compensada com suspeita de HPCS (critério não invasivo) avaliando a sobrevida (NCT03654053); STATLIVER: cirrose e GPVH maior do que $10 \mathrm{mmHg}$ avaliando a sobrevida livre de transplante e a internação hospitalar (NCT04072601) e o estudo LI VERHOPE: prevenção de ACLF em pacientes com cirrose descompensada (Child > 12) (NCT03780673).

Do que foi aqui exposto, creio ser importante levar o leitor a revisitar as consideraçôes atualizadas da Sociedade Brasileira de Hepatologia no que tange a hemorragia digestiva por ruptura de $\mathrm{VE}^{8}$, uma vez que traduzem a realidade do que está sendo feito ou do que pode ser feito em nosso País. No entanto, baseado em dados emergentes, o paradigma pode mudar, com um enfoque no tratamento da HPCS em vez de ser focado na abordagem do sangramento por varizes de alto risco e, assim, prevenir qualquer descompensaçáo (sangramento por varizes, ascite ou EH). Dentro da nova perspectiva a ser considerada, o carvedilol provavelmente seria a droga ideal a ser utilizada. Acreditamos 
que o Consenso de Baveno VII a ser realizado em outubro deste ano nos dará esta resposta. No que tange ao papel das estatinas a ser acrescido no armamentário terapêutico, creio que não poder ser considerado um mito, ou seja, apenas uma narrativa que guarda um fundo de verdade. Também não creio que hoje possa ser considerada uma realidade do tratamento das doenças hepáticas crônicas avançadas. Mas tenho convicção que, em um futuro muito próximo, após os resultados dos estudos prospectivos e randomizados em andamento, seu papel será definitivamente desnudado, e, talvez se torne mais uma ferramenta a ser utilizada na terapia dos paciente com cirrose.

\section{REFERÊNCIAS BIBLIOGRÁFICAS}

1) Nader LA, Mattos AA, Bastos GA. Burden of liver disease in Brazil. Liver International 2014; 34:844-9

2) Moon AM, Singal AG, Tapper EB. Contemporary Epidemiology of Chronic Liver Disease and Cirrhosis. Clin Gastroenterol Hepatol 2020; 18:2650-2666

3) D'Amico G, Morabito A, D'Amico M, Pasta L, Malizia G, Rebora P, Valsecchi MG. Clinical states of cirrhosis and competing risks. J Hepatol 2018; 68:563-76
4) Magaz M, Baiges A, Hernández-Gea $\mathrm{V}$. Precision medicine in variceal bleeding: Are we there yet? J Hepatol 2020; 72:774-784.

5) John JA, de Mattos AA, da Silva Miozzo SA, Comerlato PH, Porto M, Contiero P, da Silva RR. Survival and risk factors related to death in outpatients with cirrhosis treated in a clinic in Southern Brazil. Eur J Gastroenterol Hepatol. 2015;27:13727.

6) Franchis R., Expanding consensus in portal hypertension: Report of the Baveno VI Consensus Workshop: Stratifying risk and individualizing care for portal hypertension. J Hepatol 2015; 63:743-52.

7) Garcia-Tsao G, Abraldes JG, Berzigotti A, Bosch J. Portal hypertensive bleeding in cirrhosis: Risk stratification, diagnosis, and management: 2016 practice guidance by the American Association for the study of liver diseases. Hepatology 2017; 65:310-35.

8) Bittencourt PL, Strauss E, Farias AQ, Mattos AA, Lopes EP. Variceal bleeding: Update of recommendations from the Brazilian Association of Hepatology. Arq Gastroenterol. 2017; 54:349-355.

9) Mattos AZ, Schacher FC, Neto GJ, Mattos AA. Screening for esophageal 
varices in cirrhotic patients - Noninvasive methods. Ann Hepatol 2019; 18:673-678.

10) European Association for the Study of the Liver. EASL Clinical Practice Guidelines for the management of patients with decompensated cirrhosis. J Hepatol. 2018;69:406-460.

11) Jakab SS\& Garcia-Tsao G. Screening and Surveillance of Varices in Patients With Cirrhosis. Clin Gastroenterol Hepatol 2019; 17:26-29.

12) Vorobioff JD \& Groszmann JG. Prevention of portal hypertension: from variceal development to clinical decompensation. Hepatology 2015; 61:375-81.

13) Lampertico P, Invernizzi F, Viganò M, Loglio A, Mangia G, Facchettiet $\mathrm{F}$ et al. The long-term benefits of nucleos $(\mathrm{t})$ ide analogs in compensated HBV cirrhotic patients with no or small esophageal varices: A 12-year prospective cohort study. J Hepatol 2015; 63:1118-2563.

14) Lens $S$, Alvarado-Tapias E, Mariño Z, Londoño MC, LLop E, Martinez $\mathrm{J}$, et al. Effects of All-Oral AntiViral Therapy on HVPG and Systemic Hemodynamics in Patients With Hepatitis C Virus-Associated Cirrhosis. Gastroenterology 2017; 153:1273-1281.
15) Puigvehí M, Londoño MC, Torras $\mathrm{X}$, Lorente S, Vergara M, Morillas RM et al. Impact of sustained virological response with DAAs on gastroesophageal varices and Baveno criteria in HCV-cirrhotic patientsJ Gastroenterol 2020; 55:205-216.

16) Lens $S$, Baiges $A$, Alvarado-Tapias E, LLop E, Martinez J, Fortea JI et al. Clinical outcome and hemodynamic changes following HCV eradication with oral antiviral therapy in patients with clinically significant portal hypertension. Journal of Hepatology 2020;73:1415-1424.

17) Berzigotti A, Albillos A, Villanueva C, Genescá J, Ardevol A, Augustín S et al. Effects of an intensive lifestyle intervention program on portal hypertension in patients with cirrhosis and obesity: The SportDiet study. Hepatology 2017;65:1293-1305.

18) Groszmann RJ, Garcia-Tsao G, Bosch J, Grace ND, Burroughs AK, Planas $\mathrm{R}$, et al. Beta-blockers to prevent gastroesophageal varices in patients with cirrhosis. N Engl J Med. 2005 Nov 24;353(21):2254-61.

19) Villanueva C, Albillos A, Genescà J, Abraldes JG, Calleja JL, Aracil C et al. Development of hyperdynamic circulation and response to B-blockers in compensated cirrhosis with portal hypertension. Hepatology. 2016;63:197-206. 
20) Villanueva $\mathrm{C}$, Albillos A, Genescà J, Garcia-Pagan JC, Calleja JL, Aracil C et al. $B$ blockers to prevent decompensation of cirrhosis in patients with clinically significant portal hypertension (PREDESCI): a randomised, double-blind, placebocontrolled, multicentre trial. Lancet 2019;393:1597-1608.

21) Lebrec D, Poynard T, Hillon P, Benhamou JP. Propranolol for prevention of recurrent gastrointestinal bleeding in patients with cirrhosis: a controlled study. N Engl J Med. 1981;305:1371-4.

22) Turco L, Villanueva C, La Mura V, García-Pagán JC, Reiberger T, Genescà $\mathrm{J}$ et al. Lowering Portal Pressure Improves Outcomes of Patients With Cirrhosis, With or Without Ascites: A Meta-Analysis. Clin Gastroenterol Hepatol 2020;18:313-327.

23) Zacharias AP, Jeyaraj R, Hobolth L, Bendtsen F, Gluud LL, Morgan MY. Carvedilol versus traditional, nonselective beta-blockers for adults with cirrhosis and gastroesophageal varices Cochrane Database Syst Rev

24) Sharma M, Singh S, Desai V, Shah VH, Kamath PS, Murad MH, Simonetto DA. Comparison of Therapies for Primary Prevention of Esophageal Variceal Bleeding: A Systematic Review and Network Meta- analysis. Hepatology 2019;69:16571675.

25) Garcia-Tsao G, Abraldes JG. Non-selective beta-blockers in compensated cirrhosis: Preventing variceal hemorrhage or preventing decompensation? Gastroenterology (2021),doi: https://doi.org/10.1053/j. gastro.2021.04.077

26) Ioannidis JP. More than a billion people taking statins?: Potential implications of the new cardiovascular guidelines. JAMA 2014;311:463-4.

27) Pose E, Trebicka J, Mookerjee RP, Angeli P, Ginès P. Statins: Old drugs as new therapy for liver diseases? J Hepatol. 2019;70:194-202.

28) Bosch J, Gracia-Sancho J, Abraldes JG. Cirrhosis as new indication for statins. Gut 2020;69:953-962.

29) Zafra C, Abraldes JG, Turnes J, Berzigotti A, Fernández M, GarcaPagán JC et al. Simvastatin enhances hepatic nitric oxide production and decreases the hepatic vascular tone in patients with cirrhosis Gastroenterology 2004; 126:749-55.

30) Abraldes JG, Albillos A, Bañares R, Turnes J, González R, García-Pagán JC, Bosch J. Simvastatin lowers portal pressure in patients with cirrhosis and portal hypertension: a randomized controlled trial Gastroenterology 2009; 136:1651-8. 
31) Mohanty A, Tate JP, Garcia-Tsao G. Statins Are Associated With a Decreased Risk of Decompensation and Death in Veterans With Hepatitis C-Related Compensated Cirrhosis. Gastroenterology. 2016;150:430-40.

32) Bishnu S, Ahammed SM, Sarkar A, Hembram J, Chatterjee S, Das K et al. Effects of atorvastatin on portal hemodynamics and clinical outcomes in patients with cirrhosis with portal hypertension: a proof-of-concept study. Eur J Gastroenterol Hepatol 2018; 30:54-59.

33) Vijayaraghavan $R$, Jindal $A$, Arora $V$, Choudhary A, Kumar G, Sarin SK et al. Hemodynamic Effects of Adding Simvastatin to Carvedilol for Primary Prophylaxis of Variceal Bleeding: A Randomized Controlled Trial. Am J Gastroenterol 2020; 115:729-737.

34) Abraldes JG, Villanueva C, Aracil C, Turnes J, Hernandez-Guerra $\mathrm{M}$, Genesca J et al. Addition of Simvastatin to Standard Therapy for the Prevention of Variceal Rebleeding Does Not Reduce Rebleeding but Increases Survival in Patients With Cirrhosis. Gastroenterology 2016; 150:1160-1171.

35) Bakar,NS, Neely D, Avery P, Brown C, Daly AK, Kamali F. Genetic and Clinical Factors Are Associated With Statin-Related Myotoxicity of
Moderate Severity: A Case-Control Study. Clin. Pharmacol. Ther. 2018;104:178-187.

36) Theile D, Haefeli WE, Seitz HK, Millonig G, Weiss J, Mueller

S. Association of liver stiffness with hepatic expression of pharmacokinetically important genes in alcoholic liver disease. Alcohol Clin Exp Research 2013;37 Suppl 1:E17-22.

37) More VR, Cheng Q, Donepudi AC, Buckley DB, Lu ZJ, Cherrington NJ, Slitt AL. Alcohol cirrhosis alters nuclear receptor and drug transporter expression in human liver. Drug Met Disp 2013 ;41:1148-55.

38) Stokkeland K, Höijer J, Bottai M, Söderberg-Löfdal K, Bergquist A. Statin Use Is Associated With Improved Outcomes of Patients With Primary Sclerosing Cholangitis. Clin Gastroenterol Hepatol 2019; 17:1860-1866.

39) Kim RG, Loomba R, Prokop LJ, Singh $S$ et al. Statin Use and Risk of Cirrhosis and Related Complications in Patients With Chronic Liver Diseases: A Systematic Review and Meta-analysis Clin Gastroenterol Hepatol 2017; 15:1521-1530.

40) Simon TG, Duberg AS, Aleman S, Hagstrom H, Nguyen LH, Khalili $\mathrm{H}$ et al. Lipophilic Statins and Risk for Hepatocellular Carcinoma and 
Death in Patients With Chronic Viral Hepatitis: Results From a Nationwide Swedish Population. Ann Intern Med 2019; 171:318-327.

41) Pose E, Trebicka J, Mookerjee RP, Angeli P, Ginès P. Statins: Old drugs as new therapy for liver diseases? J Hepatol. 2019 ;70:194-202

42) Sung S, Al-Karaghouli M, Kalainy S, Cabrera Garcia L, Abraldes JG. A systematic review on pharmacokinetics, cardiovascular outcomes and safety profiles of statins in cirrhosis. BMC Gastroenterol 2021; 21:120 